\title{
Molecular and biochemical characterization of key enzymes in the cysteine and serine metabolic pathways of Acanthamoeba castellanii
}

\author{
Duo $\mathrm{Wu}^{1 \dagger}$, Meng Feng ${ }^{1 \dagger}$, Zhi-xin Wang ${ }^{1}$, Ke Qiao ${ }^{1}$, Hiroshi Tachibana ${ }^{2}$ and Xun-jia Cheng ${ }^{1 *}$
}

\begin{abstract}
Background: Acanthamoeba spp. can cause serious human infections, including Acanthamoeba keratitis, granulomatous amoebic encephalitis and cutaneous acanthamoebiasis. Cysteine biosynthesis and the L-serine metabolic pathway play important roles in the energy metabolism of Acanthamoeba spp. However, no study has confirmed the functions of cysteine synthase (AcCS) in the cysteine pathway and phosphoglycerate dehydrogenase (AcGDH) or phosphoserine aminotransferase (ACSPAT) in the non-phosphorylation serine metabolic pathway of Acanthamoeba.

Methods: The ACCS, AcGDH and ACSPAT genes were amplified by PCR, and their recombinant proteins were expressed in Escherichia coli. Polyclonal antibodies against the recombinant proteins were prepared in mice and used to determine the subcellular localisation of each native protein by confocal laser scanning microscopy. The enzymatic activity of each recombinant protein was also analysed. Furthermore, each gene expression level was analysed by quantitative PCR after treatment with different concentrations of cysteine or L-serine.

Results: The ACCS gene encodes a 382-amino acid protein with a predicted molecular mass of $43.1 \mathrm{kDa}$ and an isoelectric point (pl) of 8.11. The AcGDH gene encodes a 350-amino acid protein with a predicted molecular mass of $39.1 \mathrm{kDa}$ and a pl of 5.51. The ACSPAT gene encodes a 354-amino acid protein with a predicted molecular mass of $38.3 \mathrm{kDa}$ and a pl of 6 . 26. Recombinant AcCS exhibited a high cysteine synthesis activity using O-acetylserine and $\mathrm{Na}_{2} \mathrm{~S}$ as substrates. Both GDH and SPAT catalysed degradation, rather than synthesis, of serine. Exogenous L-serine or cysteine inhibited the expression of all three enzymes in a time- and dose-dependent manner.
\end{abstract}

Conclusions: This study demonstrated that ACCS participates in cysteine biosynthesis and serine degradation via the nonphosphorylation serine metabolic pathway, providing a molecular basis for the discovery of novel antiAcanthamoeba drugs.

Keywords: Cysteine synthase, Cysteine biosynthesis, Glycerate dehydrogenase, Glycolysis pathway, Drug target

\section{Background}

Free-living amoebae of the genus Acanthamoeba are widespread protozoans that exist as vegetative trophozoites and dormant cysts during their life-cycle [1]. Some Acanthamoeba species cause accidental infections, such as Acanthamoeba keratitis [2], fatal granulomatous

\footnotetext{
* Correspondence: xjcheng@shmu.edu.cn

†Duo Wu and Meng Feng contributed equally to this work.

${ }^{1}$ Department of Medical Microbiology and Parasitology, School of Basic

Medical Sciences, Fudan University, Shanghai 200032, China

Full list of author information is available at the end of the article
}

amoebic encephalitis or cutaneous infections in immunosuppressed individuals [3]. The number of reported cases worldwide is increasing every year. Despite the medical importance of these parasites, however, few antimicrobial agents are available for treatment [4].

Amino acid metabolic pathways are potential drug targets in some infectious diseases. L-cysteine is a sulphurcontaining amino acid that plays an important role in the structure, stability, catalytic activity and regulation of numerous proteins [5-9]. This amino acid is also involved in the synthesis of many other important biomolecules, such

(C) The Author(s). 2018 Open Access This article is distributed under the terms of the Creative Commons Attribution 4.0 International License (http://creativecommons.org/licenses/by/4.0/), which permits unrestricted use, distribution, and 
as glutathione, thiamine, taurine, lipoic acid, biotin and coenzyme A. Therefore, its synthetic pathways represent an attractive drug target for treatment. Cysteine can be generated from methionine through the transsulphuration pathway in mammals [5] and fungi [10] or from serine and inorganic sulphide. The latter pathway is also known as sulphur assimilation and occurs in bacteria [11], plants [12] and a few protozoa, such as Entamoeba histolytica [13] and Trypanosoma cruzi [14]. This pathway comprises two catalytic steps that are initiated by serine acetyltransferase (SAT) to form $\mathrm{O}$-acetylserine (OAS) from L-serine and acetyl-coenzyme A. OAS reacts with sulphide to generate cysteine, and this step is catalysed by cysteine synthase (CS, OAS thiolyase) [15].

To date, the presence of the sulphur assimilation/cysteine biosynthetic pathway in $A$. castellanii remains unclear. However, in a previous study, we confirmed the existence of a phosphorylated serine biosynthetic pathway in this organism [16]. Phosphoglycerate dehydrogenase (PGDH) catalyses the first step of this pathway by oxidising 3-phosphoglycerate to 3-phosphohydroxypyruvate (3-PHP) using $\mathrm{NAD}^{+} / \mathrm{NADH}$ as a cofactor. Phosphoserine aminotransferase (PSAT) catalyses the reversible conversion of 3-PHP to phosphoserine, which is then dephosphorylated by phosphoserine phosphatase to form L-serine [17]. Serine is a cysteine precursor in the sulphur assimilation/cysteine biosynthetic pathway of nearly all organisms, including protozoa. Hence, serine metabolic pathways are important in the regulation of cysteine levels.

To understand the role of serine metabolism and sulphur-containing cysteine biosynthesis in A. castellanii, we searched the Kyoto Encyclopaedia of Genes and Genomes database and created a list of potential enzymes based on the available pathway information. Then, we successfully cloned the gene of D-glycerate dehydrogenase (GDH) and serine-pyruvate aminotransferase (SPAT) from A. castellanii and demonstrated that this amoeba possesses phosphorylated and nonphosphorylated pathways for serine metabolism. We extended this work by cloning the CS gene of $A$. castellanii. However, we could not identify the gene for SAT, which is absent in this organism because no homologous genes could be found for it. This finding demonstrates that $A$. castellanii possesses a cysteine biosynthetic pathway involving CS but not SAT. Humans lack CS; thus, this parasitic enzyme could be a target for the rational design and screening of anti-Acanthamoeba agents. To the best of our knowledge, this work is the first to report on the non-phosphorylated pathway for serine metabolism and CS-mediated cysteine biosynthesis in a free-living amoeba.

\section{Methods}

\section{Chemicals}

All chemicals were of analytical grade and purchased from Sigma-Aldrich (Shanghai, China), unless stated otherwise.

\section{Amoeba culture}

Acanthamoeba castellanii (strain ATCC30011) was obtained from the American Type Culture Collection, and trophozoites were routinely grown axenically in peptoneyeast-glucose (PYG) medium [18]. Cultures were incubated at $26^{\circ} \mathrm{C}$, and trophozoites were harvested in the late $\log$ phase after subculture for $72 \mathrm{~h}$.

\section{Cloning and expression of genes}

Total A. castellanii RNA was extracted from trophozoites using RNeasy ${ }^{\circ}$ Plus Mini Kit (Qiagen, Hilden, Germany), and complementary DNA (cDNA) was synthesised using a PrimeScript ${ }^{\circ} 1$ st strand cDNA synthesis kit (Takara, Kusatsu, Japan). The CS, GDH and SPAT genes were amplified by PCR using the primers listed in Table 1. PCR was performed in a 9902 Veriti 96-well Thermal Cycler (Applied Biosystems, Waltham, USA) $\left(94{ }^{\circ} \mathrm{C}\right.$ for $3 \mathrm{~min} ; 35$ cycles of $94{ }^{\circ} \mathrm{C}$ for $15 \mathrm{~s}, 55{ }^{\circ} \mathrm{C}$ for 30 s and $72{ }^{\circ} \mathrm{C}$ for $1 \mathrm{~min}$; followed by $72{ }^{\circ} \mathrm{C}$ for $5 \mathrm{~min}$ ). The amplified PCR product was purified and ligated into a pMD19-T Vector (Takara), and the nucleotide sequence was obtained by automated sequencing. The correct plasmids were amplified with primers containing NdeI and BamHI restriction site sequence, and the PCR products were ligated into pET19b. The sequences of all constructs were confirmed on both strands and analysed with Vector NTI software (Invitrogen, Waltham, USA). Plasmids were transformed into E. coli BL21(DE3) pLysS for protein expression, and recombinant CS, GDH and SPAT proteins were purified using a QIA Express kit in accordance with the manufacturer's instructions. The purity and mass of each protein was determined by sodium dodecyl sulphate polyacrylamide gel electrophoresis. The concentrations of recombinant proteins were measured using a protein assay (Bio-Rad, Hercules, USA).

\section{Preparation of polyclonal antibodies and dot blot analysis} Each purified recombinant protein was injected intraperitoneally into BALB/c mice. In brief, $50 \mu \mathrm{g}$ of protein was mixed with Freund's complete adjuvant for the first injection, and Freund's incomplete adjuvant was used for the next two immunisations at 2-week intervals. One week after the final injection, serum was collected from the mice. Total crude protein from A. castellanii trophozoites $(5 \mu \mathrm{g})$ and aliquots of purified CS, GDH and SPAT $(1 \mu \mathrm{g})$ were blotted on a nitrocellulose membrane [19]. Recombinant Babesia microti merozoites (BMSA) [20] and antigen extracts from Dermatophagoides farinae (Derf 2; 
Table 1 Primers used for amplification of Acanthamoeba castellanii genes

\begin{tabular}{lll}
\hline Gene & Primer & Sequence (5'-3') \\
\hline CS & CS-ORF-S & ATGAAGCACATGACCCCACGC \\
& CS-ORF-AS & TTATTGGAGGTACTTGTCGGGAAGG \\
& CS-qPCR-S & CTCCGTCCTCTATTCGTAC \\
& CS-qPCR-AS & ATGCTCATTGTTCTTCGT \\
GDH & GDH-ORF-S & ATGGACAGCGGTGCGAG \\
& GDH-ORF-AS & TCAAGGATTGACGCGCATCA \\
& GDH-qPCR-S & GGTCGCATTGGCAAGAGG \\
& GDH-qPCR-AS & GCGTCGAGTCCGTGAGGTT \\
SPAT & SPAT-ORF-S & ATGGAGGCTGACCGTCCGCTGCT \\
& SPAT-ORF-AS & TTAGAGCTTGTTGGGCAGTGCTG \\
& SPAT-qPCR-S & TCGCCCGACTTTATCAACCTCT \\
& SPAT-qPCR-AS & TGCGCACCGAACGACTCCAA \\
\hline
\end{tabular}

stocks from our laboratory, $1 \mu \mathrm{g}$ ) were used as negative controls. Filter strips were blocked with $3 \%$ skimmed milk powder in PBS and then incubated with 1:50 diluted anti-AcCS, anti-AcGDH or anti-AcSPAT mouse sera. Horseradish peroxidase-conjugated (HRP) goat anti-mouse IgG (Cappel, Chester, USA) was used as a secondary antibody. Proteins were visualised using an enhanced HRP-DAB substrate detection kit (Tiangen Biotech, Beijing, China).

\section{Confocal microscopy}

Acanthamoeba castellanii trophozoites at a density of 2 $\times 10^{5}$ cells $/ \mathrm{ml}$ were used for confocal microscopy. To assess colocalisation of native $A c \mathrm{GDH}$ and AcSPAT, the trophozoites were incubated for $1 \mathrm{~h}$ with a mouse antiAcGDH polyclonal antibody (1:50 dilution with $3 \%$ skimmed milk powder in PBS), followed by $1 \mathrm{~h}$ with an Alexa Fluor 488 goat anti-mouse $\operatorname{IgG}_{1}(\mathrm{H}+\mathrm{L}$; Abcam, Cambridge, USA) as a secondary antibody. Next, an anti-AcSPAT mouse polyclonal antibody (1:50 dilution) was used to react with native AcSPAT for $1 \mathrm{~h}$, followed by an Alexa Fluor 568 goat anti-mouse $\operatorname{IgG}_{2 b}(\mathrm{H}+\mathrm{L}$; Abcam) as a secondary antibody for $1 \mathrm{~h}$. The cells were also stained with $0.25-\mathrm{mg} / \mathrm{ml}$ 4',6-diamidino-2-phenylindole (DAPI) and $1.25-\mathrm{mg} / \mathrm{ml}$ 1,4-diazabicyclo[2.2.2]octane (DABCO, in $10 \%$ glycerol-PBS) for $10 \mathrm{~min}$. For AcCS localisation, the cells were incubated for $1 \mathrm{~h}$ with anti-AcCS mouse polyclonal antibody (1:50 dilution) used as a primary antibody, followed by $1 \mathrm{~h}$ with an Alexa Fluor 488 goat anti-mouse $\operatorname{IgG}_{1}(\mathrm{H}+\mathrm{L}$; Abcam $)$ used as a secondary antibody. Propidium iodide was used to stain nuclei. Cell suspensions containing $2 \times 10^{4}$ cells were placed onto glass slides, mounted with coverslips and examined using a Leica TCS SP8 (Wetzlar, Germany) microscope.

\section{Cysteine synthase assay}

CS activity was measured as described by Westrop et al. [21]. Briefly, reaction mixtures contained $50 \mathrm{mM}$ Tris- $\mathrm{HCl}$ (pH 7.5), $0.2 \mathrm{mM}$ pyridoxal 5'-phosphate, 20 $\mathrm{mM}$ OAS, $2 \mathrm{mM} \mathrm{Na}_{2} \mathrm{~S}$ and the corresponding recombinant CS protein. Reactions were stopped by adding 50 $\mu \mathrm{l}$ of glacial acetic acid (> 99.9\%), and the formation of cysteine was quantified by the method described by Gaitonde [22]. The $\mathrm{K}_{\mathrm{m}}$ for OAS was determined using 3 $\mathrm{mM} \mathrm{Na} \mathrm{N}_{2} \mathrm{~S}$ and by varying the concentration of OAS between 10 and $100 \mathrm{mM}$. The $\mathrm{K}_{\mathrm{m}}$ of $\mathrm{Na}_{2} \mathrm{~S}$ was determined using $30 \mathrm{mM}$ OAS and by varying the concentrations of $\mathrm{Na}_{2} \mathrm{~S}$ between 0.1 and $12.8 \mathrm{mM}$.

\section{Glycerate dehydrogenase assay}

GDH activity was determined in both forward and reverse directions [13]. Briefly, the reactions contained 20 $\mathrm{mM}$ sodium phosphate buffer ( $\mathrm{pH} 6.5), 300 \mathrm{mM} \mathrm{NaCl}$, $0.2 \mathrm{mM}$ NADPH, $0.2 \mathrm{mM}$ dithiothreitol (DTT), $10 \mathrm{mM}$ lithium $\beta$-hydroxypyruvic acid (hydroxypyruvate, HP) and the purified protein. The $\mathrm{K}_{\mathrm{m}}$ for HP was determined using $1.4 \mathrm{mM} \mathrm{NADPH}$ and by varying the concentration of HP between 7.5 and $90 \mathrm{mM}$. The $\mathrm{K}_{\mathrm{m}}$ of NADPH was determined using $22.5 \mathrm{mM} \mathrm{HP}$ and by varying the concentrations of NADPH between 0.4 and $8 \mathrm{mM}$. In the reverse reactions, the reactions contained $50 \mathrm{mM}$ Tris- $\mathrm{HCl}$ buffer (pH 8.5), $300 \mathrm{mM} \mathrm{NaCl}, 0.2 \mathrm{mM}$ DTT, $10 \mathrm{mM} \mathrm{NADP}^{+}, 10 \mathrm{mM}$ hemicalcium D-glyceric acid (glycerate) and the protein. The $\mathrm{K}_{\mathrm{m}}$ for glycerate was determined using $30 \mathrm{mM} \mathrm{NADP}^{+}$and by varying the concentration of glycerate between 100 and $1000 \mathrm{mM}$. The $\mathrm{K}_{\mathrm{m}}$ of $\mathrm{NADP}^{+}$was determined using $150 \mathrm{mM}$ glycerate and by varying the concentrations of $\mathrm{NADP}^{+}$between 20 and $240 \mathrm{mM}$.

\section{Serine-pyruvate aminotransferase assay}

Serine-pyruvate aminotransferase activity was detected as described by Snell et al. [23]. Briefly, reaction mixtures contained $50 \mathrm{mM}$ Tris- $\mathrm{HCl}$ buffer $(\mathrm{pH} 8.5), 20$ $\mathrm{mM}$ L-serine, $20 \mathrm{mM}$ sodium pyruvate and $40 \mu \mathrm{M}$ pyridoxal 5'-phosphate (PLP), and the reaction was initiated by adding purified SPAT protein. After incubation for 30 min, $70 \%(w / v) \mathrm{HClO}_{4}$ was added to stop the reactions. Subsequently, active $A c \mathrm{GDH}$ proteins were used to assess HP generation. The $K_{m}$ for serine was determined using $4 \mathrm{mM}$ pyruvate and by varying the concentration of serine between 0.125 and $5 \mathrm{mM}$. The $\mathrm{K}_{\mathrm{m}}$ of pyruvate was determined using $0.5 \mathrm{mM}$ serine and by varying the concentration of pyruvate between 2 and $25 \mathrm{mM}$.

The kinetic constants reported for all assays are the means of at least three independent determinations, and $K_{m}$ and $\mathrm{V}_{\max }$ values were estimated using Michaelis-Menten and Lineweaver-Burk plots in conjunction with non-linear regression using GraphPad Prism v.5.01. Turnover numbers 
$\left(k_{\text {cat }} s^{-1}\right)$ were calculated using the molecular mass of one subunit of each protein.

\section{Treatment with L-serine or cysteine and quantitative PCR} analysis

Trophozoites were seeded in a 24-well plate (Eppendorf, Hamburg, Germany) at a density of $1 \times 10^{5} \mathrm{cells} / \mathrm{ml}$ in PYG medium [24]. L-serine or cysteine were added at concentrations of 10, 20 and $40 \mathrm{mM}$, followed by incubation at $26^{\circ} \mathrm{C}$. Trophozoites were collected after 12 and 24 $\mathrm{h}$ of incubation, and total RNA was extracted. Real-time quantitative PCR was performed to analyse $A c \mathrm{CS}, A c \mathrm{GDH}$ and $A c S P A T$ gene expression. The primers used are shown in Table 1.

\section{Statistical analysis}

All statistical analysis was performed using GraphPad 5 software (San Diego, CA, USA). Significance was calculated by one-way ANOVA analysis followed by a Tukey test. Data were expressed as the mean \pm standard deviation (SD) and at least three independent experiments were performed for each sample. $P<0.05$ was considered to be significant.

\section{Results}

Cloning of the genes encoding $A$. castellanii CS, GDH and SPAT

The sequences of GDH and SPAT with the accession numbers LC154947 and LC154948, respectively, have been submitted to the GenBank database, whereas the sequence of CS is still under submission. The CS gene contains a 1149-bp open reading frame (ORF) that encodes a 382-amino acid protein with a predicted molecular mass of $43.1 \mathrm{kDa}$ and an isoelectric point ( $\mathrm{pI}$ ) of 8.11. The GDH gene contains a 1053-bp ORF that encodes a 350-amino acid protein with a predicted molecular mass of $39.1 \mathrm{kDa}$ and a pI of 5.51. The SPAT gene contains a 1062-bp ORF encoding a 354-amino acid protein with a predicted molecular mass of 38.3 $\mathrm{kDa}$ and a pI of 6.26.

\section{Reactivity of mouse polyclonal antibodies to AcCS, AcGDH and ACSPAT}

The reactivities of polyclonal antibodies raised against AcCS, AcGDH and AcSPAT were assessed by a dot blot analysis (Fig. 1). Crude (5 $\mu \mathrm{g})$ and recombinant proteins (1 $\mu \mathrm{g})$ were spotted on a nitrocellulose membrane, and the crude antigens in strips 1, 2 and 3 exhibited strong immunoreactivity, indicating that the polyclonal antibodies recognised the respective proteins in trophozoites. However, the anti-AcSPAT polyclonal antibody exhibited cross-reactivity with both $\mathrm{CS}$ and GDH recombinant proteins. No immunoreactivity was observed in normal mouse serum to any of the spotted antigen.

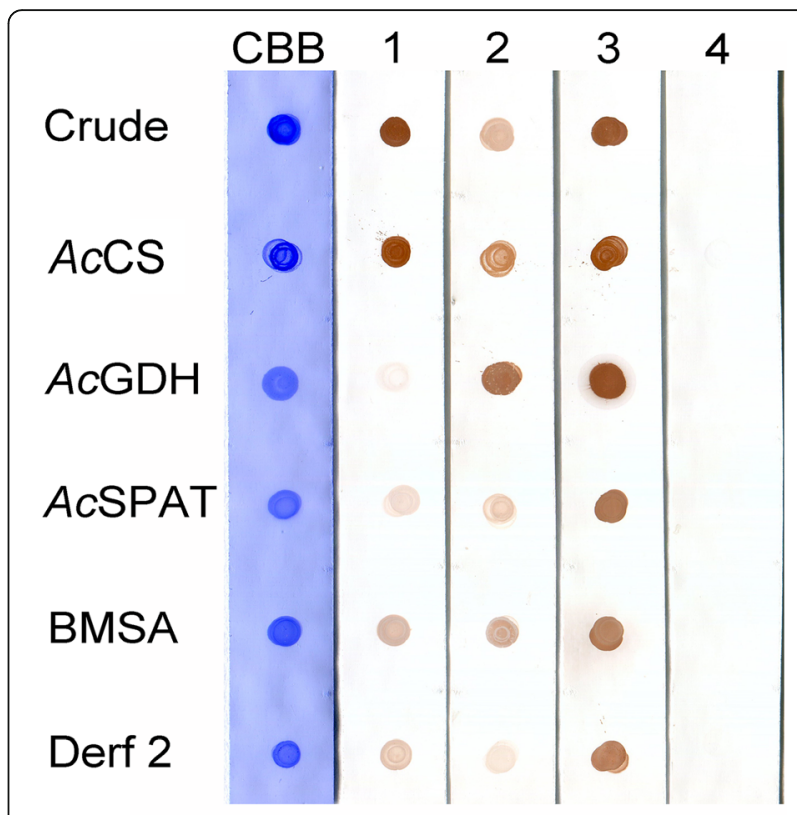

Fig. 1 Dot blot analysis of reactivity of mouse polyclonal antibodies to $A c C S, A c G D H$ or AcSPAT. Strips were spotted with extracted crude proteins from trophozoites $(5 \mu \mathrm{g})$ and with each purified recombinant protein $(1 \mu \mathrm{g})$. BMSA and Derf 2 were used as negative controls. One strip was stained with Coomassie brilliant blue. Strips 1, 2 and 3 were treated with anti-AcCS, anti-AcGDH and anti-AcSPAT mouse polyclonal serum, respectively. Strip 4 was treated with normal mouse serum as a negative control

Localisation of native CS, GDH and SPAT in A. castellanii To determine the localisation of native CS, GDH and SPAT in A. castellanii, confocal laser scanning microscopy was performed using anti-CS, anti-GDH and anti-SPAT mouse polyclonal antibodies (Fig. 2). AcCS was found to be localised to the inner part of the cell membrane (Fig. 2b), AcGDH and AcSPAT appeared to be colocalised (Fig. 2i) to the inner part of the cell membrane and to the membranes surrounding the nucleus.

\section{Kinetic properties of AcCS, AcGDH and AcSPAT}

To assess the potential role of CS in cysteine biosynthesis, its sulphydrylase activity was tested. Recombinant AcCS could readily catalyse cysteine synthesis using OAS and $\mathrm{Na}_{2} \mathrm{~S}$ as substrates and showed a high affinity for both substrates (Fig. 3). The enzyme was highly active in the $\mathrm{pH}$ range of 7.0 to 7.5 .

The $A c \mathrm{GDH}$ activity was measured in both forward and reverse directions. In the forward reaction, GDH catalyses the reduction of HP to glycerate using NADPH as a cofactor, whereas in the reverse reaction, it converts glycerate to $\mathrm{HP}$ using $\mathrm{NADP}^{+}$as a cofactor. Recombinant AcGDH exhibited a higher affinity for HP than for glycerate as $\mathrm{K}_{\mathrm{m}}$ for glycerate was 10-fold higher than $K_{m}$ for HP (Table 2). Concordantly, the apparent $\mathrm{K}_{\mathrm{m}}$ of $\mathrm{NADP}^{+}$was higher than $\mathrm{K}_{\mathrm{m}}$ of NADPH; however, the specific activity was 10 -fold 

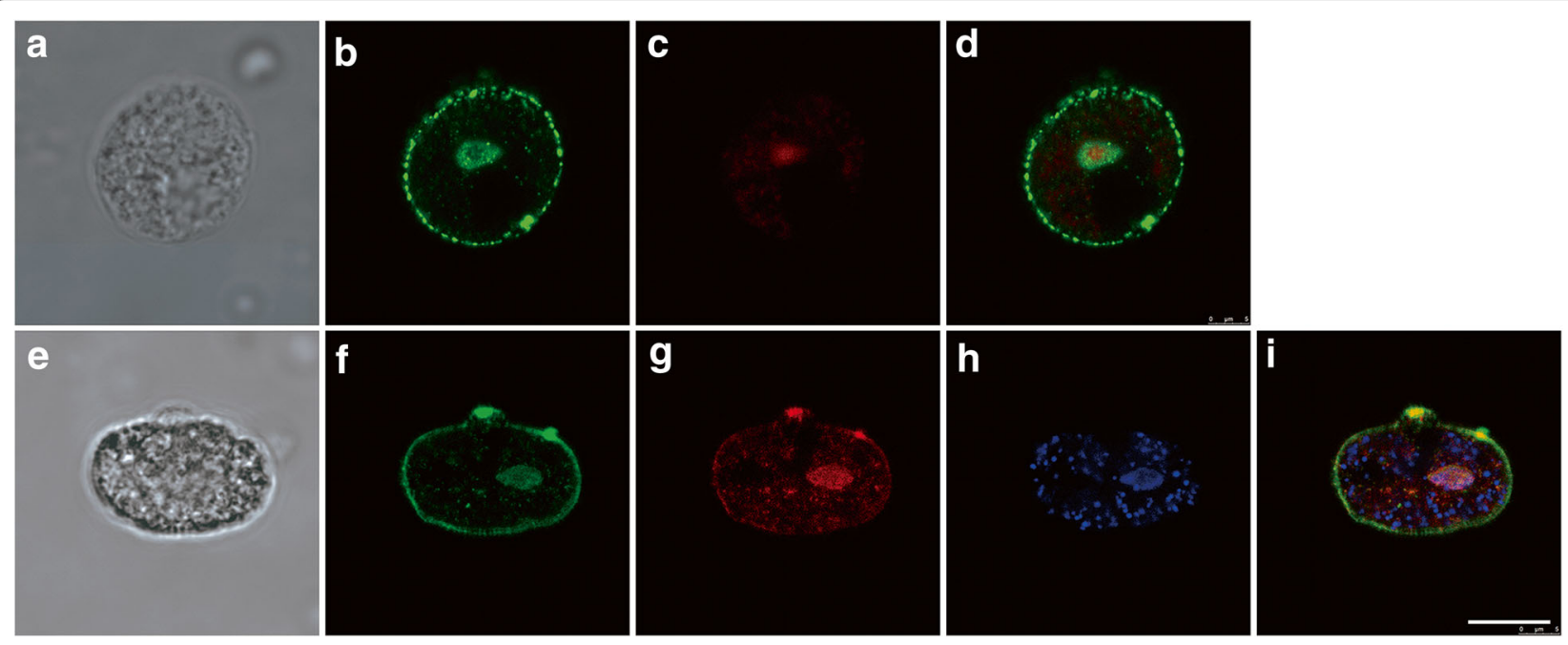

Fig. 2 Localization of native CS, DGH and SPAT in Acanthamoeba trophozoites by confocal microscopy. a-d Localisation of AcCS, e-i colocalisation of $A c G D H$ and ACSPAT in trophozoites. $\mathbf{a}$, e Differential interference contrast microscopy. $\mathbf{b}$ Staining with a polyclonal antibody against AcCS. $\mathbf{c}$ Nuclear staining with propidium iodide. $\mathbf{d}$ Merged image of $\mathbf{b}$ and $\mathbf{c}$. $\mathbf{f}$ Staining with a polyclonal antibody against AcGDH. $\mathbf{g}$ Staining with a polyclonal antibody against AcSPAT. $\mathbf{h}$ Nuclear staining with DAPI. i Merged image of $\mathbf{f}, \mathbf{g}$ and $\mathbf{h}$. Scale-bar: $10 \mu \mathrm{m}$

higher with $\mathrm{NADP}^{+}$than with NADPH. In the forward reaction, $A c \mathrm{GDH}$ exhibited a high activity in the $\mathrm{pH}$ range of 6.0-6.5, and in the reverse reaction, $A c \mathrm{GDH}$ showed the highest activity in the $\mathrm{pH}$ range of 7.8-8.5. These data suggest that $A c \mathrm{GDH}$ specifically catalyses the conversion $\mathrm{HP}$ to glycerate.

SPAT catalyses the conversion of pyruvate to HP using $\mathrm{L}$-serine as an amine donor; in the reverse reaction, it converts HP to pyruvate using alanine as an amine donor. However, we could not test for the conversion of HP to pyruvate because the addition of lactate dehydrogenase would be required to monitor HP generation. The optimal $\mathrm{pH}$ for AcSPAT was 8.0-8.5, and its specific activity was 6.99 and $13.17 \mathrm{mmol} / \mathrm{min} / \mathrm{mg}$ protein with pyruvate and serine, respectively, suggesting that AcSPAT could transform both serine and pyruvate.

\section{Effects of cysteine and L-serine on the gene expression} levels of key enzymes

To obtain further evidence that $A c C S$ is involved in cysteine biosynthesis and to verify that $A c \mathrm{GDH}$ and AcSPAT participate in the serine degradation pathway in A. castellanii, exogenous cysteine or serine were added to the culture medium of trophozoites and subsequent gene expression levels of these enzymes were assessed using quantitative PCR (Fig. 4). AcCS, AcGDH and AcSPAT expression was significantly downregulated by cysteine in a dose-dependent manner after treatment for 12 or $24 \mathrm{~h}$, and no significant difference in their expression was observed between these two time points. AcCS and AcGDH expression was also significantly inhibited by L-serine in a dose-dependent manner after incubation for 12 or $24 \mathrm{~h}$. However, AcSPAT expression slightly increased at treatment
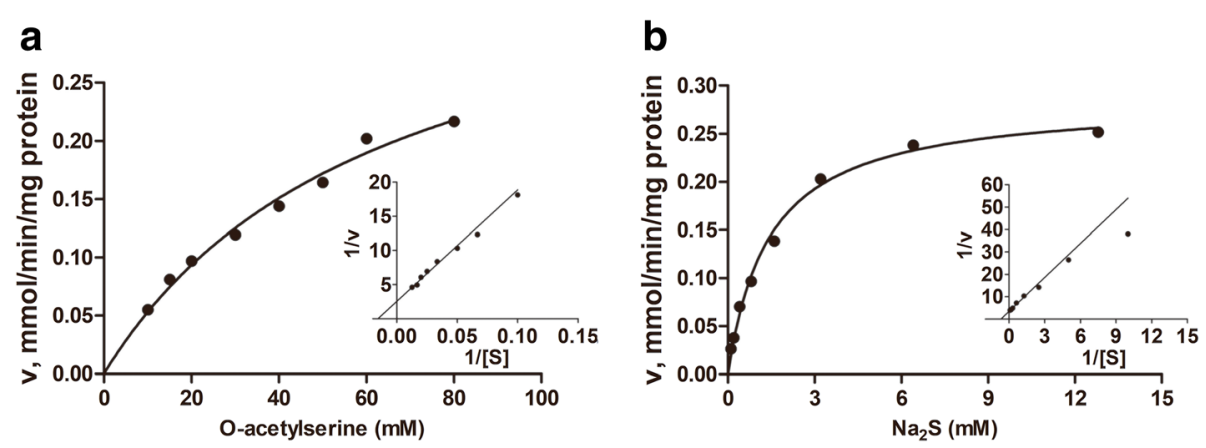

Fig. 3 Michaelis-Menten and Lineweaver-Burk double-reciprocal plots for the substrates of CS of A. castellanii. The enzyme assay was performed by varying one substrate concentration in the presence of a saturating concentration of the other substrate (see the experimental section). Panels $\mathbf{a}$ and $\mathbf{b}$ show the Michaelis-Menten plots obtained for cysteine and $\mathrm{Na}_{2} \mathrm{~S}$ as the variable substrates, respectively. The corresponding LineweaverBurk plots are depicted as insets on the right side of each panel 
Table 2 Kinetic parameters of AcCS, AcGDH and AcSPAT

\begin{tabular}{|c|c|c|c|c|}
\hline Recombinant proteins & Substrate/cofactor & $\mathrm{Km}(\mathrm{mM})$ & $\begin{array}{l}\text { Specific activity (mmol/min } \\
\text { per mg of protein) }\end{array}$ & kcat ('s) \\
\hline \multirow[t]{2}{*}{$\overline{A c C S}$} & O-acetylserine & $0.39 \pm 0.02$ & $63.92 \pm 6.33$ & \\
\hline & $\mathrm{Na}_{2} \mathrm{~S}$ & $1.44 \pm 0.08$ & $0.28 \pm 0.01$ & $2.36 \times 10^{4} \pm 978$ \\
\hline \multirow[t]{4}{*}{$A c G D H$} & Hydroxypyruvate & $17.93 \pm 1.71$ & $26.50 \pm 0.85$ & \\
\hline & $\mathrm{NADPH}$ & $1.01 \pm 0.06$ & $2.05 \pm 0.04$ & $9.5 \times 10^{3} \pm 297$ \\
\hline & Glycerate & $213.80 \pm 19.77$ & $20.48 \pm 0.65$ & \\
\hline & NADP+ & $19.90 \pm 0.74$ & $20.85 \pm 0.17$ & $1.38 \times 10^{4} \pm 273$ \\
\hline \multirow[t]{2}{*}{ ACSPAT } & Pyruvate & $5.26 \pm 0.40$ & $6.98 \pm 0.18$ & \\
\hline & Serine & $0.72 \pm 0.03$ & $13.17 \pm 0.19$ & $6.66 \times 10^{3} \pm 122$ \\
\hline
\end{tabular}

The enzyme assays were performed by varying one substrate concentration in the presence of a saturating concentration of the other substrate (see the experimental section). The $\mathrm{k}_{\mathrm{cat}}$ values reported are the means calculated from the $\mathrm{V}_{\text {max }}$ values obtained for both substrates for each enzyme (the $\mathrm{k}_{\text {cat }}$ for $A c C S$ was obtained using OAS and $\mathrm{Na}_{2} \mathrm{~S}$; the $\mathrm{k}_{\text {cat }}$ for the forward reaction of $A c G D H$ was obtained using hydroxypyruvate and NADPH, whereas that for the reverse reaction was obtained using glycerate and $\mathrm{NADP}^{+}$; the $\mathrm{k}_{\text {cat }}$ for AcSPAT was obtained using pyruvate and serine). Reactions were performed at $37{ }^{\circ} \mathrm{C}$ and results represent means \pm SD from triple experiments

with $10 \mathrm{mM}$ serine for $12 \mathrm{~h}$ but decreased at higher concentrations or after $24 \mathrm{~h}$ of treatment.

\section{Discussion}

This study is the first to report on the cysteine synthetic and non-phosphorylated serine metabolic pathways in $A$. castellanii. The properties of AcCS presented here provide molecular insights into cysteine biosynthesis in Acanthamoeba. The data showed that recombinant CS actively catalyses the generation of cysteine (Fig. 3) and that the enzyme mRNA level is substantially downregulated when trophozoites were treated by abundant exogenous cysteine treated (Fig. 4 a, d). These results demonstrate that AcCS participates in the synthesis of cysteine in Acanthamoeba. Sequence comparison (Additional file 1: Figure S1) revealed that $A c C S$ possesses all of the conserved substrate-binding sites but lacks the $\beta 8-\beta 9$ surface loop, a domain that is conserved in the CS associated with SAT [25-27]. This finding reveals that $A c C S$ does not form a regulatory complex with SAT in Acanthamoeba and is consistent with the lack of any evidence of a SAT homologue gene in the A. castellanii genome.

SPAT catalyses the first step of the non-phosphorylated serine metabolic pathway by transforming pyruvate to HP using serine as an amine donor [28]. GDH then catalyses $\mathrm{HP}$ reduction to glycerate, and glycerate kinase uses ATP as a phosphate donor to phosphorylate glycerate and produce 2-phosphoglycerate, which then enters the glycolytic pathway [29]. In the present study, we demonstrated that A. castellanii possesses SPAT and GDH in the non-phosphorylated serine metabolic pathway. The purified AcSPAT had a specific activity of $13.17 \pm 0.19 \mathrm{mmol} / \mathrm{min}$ per mg of protein for serine and showed a low $\mathrm{Km}$ when pyruvate was used as the substrate (Table 2). Such results indicate that AcSPAT actively degrades L-seine to HP in $A$. castellanii. Moreover, the apparent $K \mathrm{~m}$ of $\mathrm{D}$-glycerate and $\mathrm{NADP}^{+}$was higher than that of $\mathrm{HP}$ and $\mathrm{NADPH}$, indicating the strong partiality of the GDH of this amoeba toward HP and NADPH as substrates compared with $\mathrm{D}$-glycerate and $\mathrm{NADP}^{+}$. These data reveal that GDH may prefer to degrade, rather than generate, serine, consistent with the role of EhGDH in E. histolytica [13]. AcGDH and AcSPAT colocalise in the inner part of the cell membrane and in the membranes surrounding the nucleus (Fig. 2), thus suggesting that they have similar physiological functions and that they can enter the nucleus of Acanthamoeba to participate in metabolic reactions. A parallel phenomenon, in which plant-like glycolytic enzymes can enter the nucleus, has been observed in Toxoplasma gondii [30].

The presence of the cysteine synthetic and non-phosphorylated serine metabolic pathways indicates that these protozoa have a high requirement for cysteine, perhaps for growth, attachment and pathogenicity, similar to its functions in E. histolytica [31] and T. cruzi [14]. In contrast to E. histolytica and T. cruzi, protozoans such as Giardia intestinalis, Plasmodium falciparum and Cryptosporidium parvum [6], lack both SAT and CS, perhaps because they do not have such a high requirement for cysteine or can absorb sufficient amounts of the amino acid from their hosts. Since different physiological functions exist in the cysteine metabolism pathway of protozoans, this metabolic pathway has been considered to be a potential drug target in some infectious diseases. For instance, in Trichomonas vaginalis and E. histolytica, CS is essential for cysteine synthesis from sulphide, and this parasitic enzyme could be an exploitable drug target because humans lack CS and the sulphur assimilation/cysteine biosynthetic pathway is absent from mammalian hosts [21, 31]. In T. brucei, an inhibitor of S-adenosylmethionine decarboxylase, was found to be highly effective against infections in mice [6]; methionine adenosyltransferase is also used therapeutically in T. brucei rhodesiense infections [32]. Moreover, Plasmodium S-adenosylhomocysteine hydrolase 

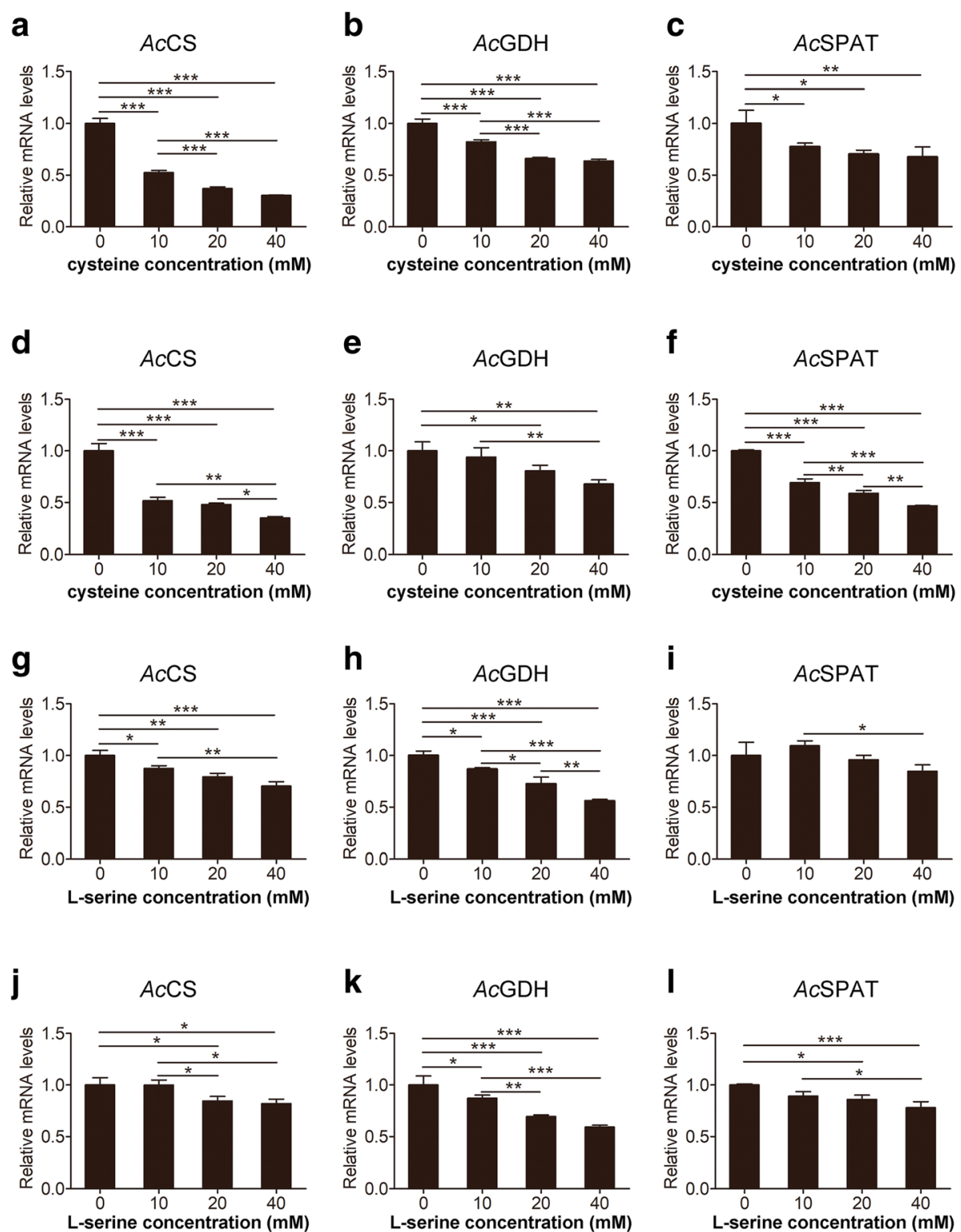

Fig. 4 Effect of cysteine (a-f) or L-serine ( $\mathbf{g}-\mathbf{I})$ on CS, GDH and SPAT gene expression in A. castellanii trophozoites. a-c Gene expression levels after treatment with different concentrations of cysteine (10, 20 and $40 \mathrm{mM})$ for $12 \mathrm{~h}$. $\mathbf{d}$-f Gene expression levels after treatment with cysteine for $24 \mathrm{~h}$. $\mathbf{g}$-i Gene expression levels after treatment with different concentrations of L-serine (10, 20 and $40 \mathrm{mM}$ ) for $12 \mathrm{~h}$. j-I Gene expression levels after treatment with serine for $24 \mathrm{~h}$. As a control, trophozoites were cultured in PYG medium with no amino acid treatment. Vertical bars indicate SD. ${ }^{*} P<0.05,{ }^{* *} P<0.01$ and ${ }^{* *} P<0.001$ by one-way ANOVA

provides a practical target for antimalarial drugs [33]. However, in-depth investigations should be performed to confirm whether the cysteine synthetic or non-phosphorylated serine metabolic pathways could also be novel targets for the development of new drugs against acanthamoebiasis.

Unlike parasitic protozoa, free-living amoebae must immediately adapt to changing environmental conditions, such as starvation, increased osmolarity, extreme $\mathrm{pH}$ or temperature and oxygen depletion [2, 34]. Physiological features may be ascribed to the complex energy metabolism of trophozoites and cysts. Acanthamoeba trophozoites feed as aerobic protozoa in the presence of sufficient oxygen; however, under anoxic conditions, many free-living protists switch between aerobic and anaerobic metabolism [35]. A. castellanii possesses a complex energetic and respiratory system in which glycolysis [36] associated with aerobic mitochondria [37] and a hydrogenosomal-type anaerobic ATP generation pathway [38] function together to maintain its energy requirements. Moreover, A. castellanii prefers the anaerobic pathway for energy production [39], thus 
indicating that glycolysis may be an important energy metabolic pathway in Acanthamoeba. However, glycolysis does not always function throughout the life-cycle of Acanthamoeba. Fructose-bisphosphate aldolase and enolase are key enzymes in glycolysis that are repressed during Acanthamoeba encystation [40], which suggests that glycolytic enzymes are expressed stage-specifically. The same phenomenon has been observed in Toxoplasma, where enolase 1 is expressed in bradyzoites and enolase 2 is expressed in tachyzoites [41]. In E. histolytica, glucose6-phosphate and fructose-6-phosphate are substantially depleted during encystation [42]. In our previous study, AcPGDH and AcPSAT were also expressed stage-specifically [16]. These enzymes were downregulated after treatment with L-serine or cysteine (Additional file 2: Figure S2), consistent with the AcCS, AcGDH and AcSPAT expression observed in this study. Serine, in conjunction with pyruvate, is located at the glycolysis gateway [13]. Hence, we hypothesise that $A c \mathrm{GDH}$ and $A c \mathrm{SPAT}$ or $A c \mathrm{CS}$ are involved in glycolysis regulation in A. castellanii.

Acanthamoeba castellanii hydrolyses glucose into pyruvate via glycolysis [34]. Glucose is a major precursor for cellulose synthesis [36], and many studies have demonstrated that cellulose synthesis is essential for cyst formation [43]. Thus, determining the role of glycolysis in cyst formation in A. castellanii is interesting [40, 44]. Analysis based on gene knockdown technology may provide an enhanced understanding of the roles of key enzymes, namely $A c \mathrm{GDH}, A c \mathrm{SPAT}$ or $A c \mathrm{CS}$, during cyst formation. This knowledge may also provide an opportunity to explore drug targets, particularly the cyst formation of $A$. castellanii.

The pathogenicity of Acanthamoeba is related to its extracellular protease activities, and serine and cysteine proteases are the predominant extracellular proteases. Several serine and cysteine proteases have been identified in collagen degradation and corneal stroma invasion [2, 45]. Cysteine and serine are the catalytic residues at the active sites of these enzymes, and the influence of the activities of these enzymes on serine or cysteine biosynthesis has yet to be clarified. These observations, along with the fact that mammals do not perform sulphur assimilation/ cysteine biosynthesis, indicate that the cysteine synthetic or nonphosphorylated serine metabolic pathway could be an attractive drug target for acanthamoebiasis treatment.

\section{Conclusions}

The gene sequences of $A c \mathrm{CS}, A c \mathrm{GDH}$ and $A c \mathrm{SPAT}$ were elucidated in this study. AcCS was involved in cysteine biosynthesis, whereas $A c \mathrm{GDH}$ and AcSPAT were involved in the non-phosphorylated serine degradation pathway and, therefore, in the glycolytic pathway of $A$. castellanii. This study is the first to provide molecular insights into the cysteine biosynthesis and non-phosphorylated serine metabolic pathways in free-living amoeba. Our results indicate the existence of the sulphur assimilation/cysteine biosynthetic pathway in the cysteine metabolism of $A$. castellanii and that AcGDH and AcSPAT may be involved in the energy metabolism, cyst formation and pathogenesis of Acanthamoeba. These findings could be further confirmed by studies using gene knockdown approaches or enzyme inhibitors. In summary, our data collectively suggest that $A c C S$ can be a target for the rational design of anti-Acanthamoeba drugs.

\section{Additional files}

Additional file 1: Figure S1. Multiple alignments of amino acid sequences of CS from A. castellanii and other representative organisms. Sequences were aligned using AlignX (Vector NTI 11.5.3, Invitrogen). Sequences are as follows: Ac, A. castellanii AcCS (this study); Bm, (Balamuthia mandrillaris, LEOU01001036); Dd, (Dictyostelium discoideum, XP629379); At (Arabidopsis thaliana, P47998); Eh (E. histolytica, BAA21916); Ei, (Entamoeba invadens, BAN42435); Lm (Leishmania major, CAJ09322); St (Salmonella typhimurium, AGQ86853); Tv (Trichomonas vaginalis, XP001325874). The letter $X$ represents the uncertain sequences of Dictyostelium discoideum CS. Black shading indicates conserved amino acids. Arrowhead indicates active site lysine. Dots indicate binding sites for sulphur incorporation into cysteine. Arrows indicate key OAS-binding sites. Underlined domains indicate $\beta 8-\beta 9$ residues of CS that interact with SAT in A. thaliana and S. typhimurium. (TIF $6729 \mathrm{~kb}$ )

Additional file 2: Figure S2. Effect of cysteine on PGDH and PSAT gene expression in A. castellanii trophozoites. $\mathbf{a}$, $\mathbf{b}$ Gene expression levels after treatment with different concentrations of cysteine $(10,20$ and $40 \mathrm{mM})$ for 12 h. c, d Gene expression levels after treatment with cysteine for 24 $\mathrm{h}$ as a control, trophozoites were cultured in PYG medium without treatment. Vertical bars indicate SD. ${ }^{*} P<0.05,{ }^{*} P<0.01$ and ${ }^{* *} P<0.001$ by one-way ANOVA. (TIF $1191 \mathrm{~kb}$ )

\section{Abbreviations}

3-PHP: 3-phosphohydroxypyruvate; BMSA: Babesia microti merozoites; CS: Cysteine synthase; GDH: D-glycerate dehydrogenase;

HP: Hydroxypyruvate; PGDH: Phosphoglycerate dehydrogenase; PSAT: Phosphoserine aminotransferase; SAT: Serine acetyltransferase; SPAT: Serine-pyruvate aminotransferase

\section{Acknowledgements}

The authors wish to thank Jun-long Cai for helping us purchase reagents used in the study.

\section{Funding}

This work was supported by the National Natural Science Foundation of China grant number 81572020 and by JSPS KAKENHI grant number JP16H05819.

\section{Availability of data and materials}

All data generated or analysed during this study are included in this published article.

\section{Authors' contributions}

DW and XJC conceived and designed the study. DW performed the experiments and wrote the paper. MF performed image capture and sequence alignment. MF and HT helped with the study design. ZXW collected materials. XJC (and HT) revised the manuscript. All authors read and approved the final manuscript.

Ethics approval and consent to participate

This study was approved by the Institutional Animal Care and Use Committee of Fudan University, China (permit numbers: 201202019 and 20150119-112). 


\section{Consent for publication}

Not applicable.

\section{Competing interests}

The authors declare that they have no competing interests.

\section{Publisher's Note}

Springer Nature remains neutral with regard to jurisdictional claims in published maps and institutional affiliations.

\section{Author details}

${ }^{1}$ Department of Medical Microbiology and Parasitology, School of Basic Medical Sciences, Fudan University, Shanghai 200032, China. ${ }^{2}$ Department of Infectious Diseases, Tokai University School of Medicine, Isehara, Kanagawa 259-1193, Japan.

\section{Received: 14 August 2018 Accepted: 6 November 2018}

\section{Published online: 26 November 2018}

\section{References}

1. Lorenzo-Morales J, Martin-Navarro CM, Lopez-Arencibia A, Arnalich-Montiel F, Pinero JE, Valladares B. Acanthamoeba keratitis: an emerging disease gathering importance worldwide? Trends Parasitol. 2013;29:181-7.

2. Lorenzo-Morales J, Khan NA, Walochnik J. An update on Acanthamoeba keratitis: diagnosis, pathogenesis and treatment. Parasite. 2015;22:10.

3. Marciano-Cabral F, Cabral G. Acanthamoeba spp. as agents of disease in humans. Clin Microbiol Rev. 2003;16:273-307.

4. Deng Y, Ran W, Man S, Li X, Gao H, Tang W, et al. Artemether exhibits amoebicidal activity against Acanthamoeba castellanii through inhibition of the serine biosynthesis pathway. Antimicrob Agents Chemother. 2015;59: 4680-8.

5. Stipanuk MH, Dominy JE Jr., Lee Jl, Coloso RM. Mammalian cysteine metabolism: new insights into regulation of cysteine metabolism. J Nutr. 2006:136(Suppl):1652S-1659S.

6. Nozaki T, Ali V, Tokoro M. Sulfur-containing amino acid metabolism in parasitic protozoa. Adv Parasitol. 2005:60:1-99.

7. Giles NM, Giles GI, Jacob C. Multiple roles of cysteine in biocatalysis. Biochem Biophys Res Commun. 2003;300:1-4.

8. Giles Gl, Tasker KM, Jacob C. Hypothesis: the role of reactive sulfur species in oxidative stress. Free Radic Biol Med. 2001;31:1279-83.

9. Jensen KS, Hansen RE, Winther JR. Kinetic and thermodynamic aspects of cellular thiol-disulfide redox regulation. Antioxid Redox Signal. 2009;11:1047-58.

10. Brzywczy J, Sienko M, Kucharska A, Paszewski A. Sulphur amino acid synthesis in Schizosaccharomyces pombe represents a specific variant of sulphur metabolism in fungi. Yeast. 2002;19:29-35.

11. Liu M, Prakash C, Nauta A, Siezen RJ, Francke C. Computational analysis of cysteine and methionine metabolism and its regulation in dairy starter and related bacteria. J Bacteriol. 2012;194:3522-33.

12. Noji M, Inoue K, Kimura N, Gouda A, Saito K. Isoform-dependent differences in feedback regulation and subcellular localization of serine acetyltransferase involved in cysteine biosynthesis from Arabidopsis thaliana. J Biol Chem. 1998:273:32739-45.

13. Ali $V$, Shigeta $Y$, Nozaki T. Molecular and structural characterization of $\mathrm{NADPH}$-dependent d-glycerate dehydrogenase from the enteric parasitic protist Entamoeba histolytica. Biochem J. 2003;375:729-36.

14. Nozaki T, Shigeta Y, Saito-Nakano Y, Imada M, Kruger WD. Characterization of transsulfuration and cysteine biosynthetic pathways in the protozoan hemoflagellate, Trypanosoma cruzi. Isolation and molecular characterization of cystathionine beta-synthase and serine acetyltransferase from Trypanosoma. J Biol Chem. 2001;276:6516-23.

15. Jeelani G, Sato D, Soga T, Watanabe H, Nozaki T. Mass spectrometric analysis of L-cysteine metabolism: physiological role and fate of L-cysteine in the enteric protozoan parasite Entamoeba histolytica. mBio. 2014:5:e01995.

16. Deng Y, Wu D, Tachibana H, Cheng X. Molecular cloning and expression of phosphoglycerate dehydrogenase and phosphoserine aminotransferase in the serine biosynthetic pathway from Acanthamoeba castellanii. Parasitol Res. 2015:114:1387-95.

17. Basurko MJ, Marche M, Darriet M, Cassaigne A. Phosphoserine aminotransferase, the second step-catalyzing enzyme for serine biosynthesis. IUBMB Life. 1999;48:525-9.
18. Garate M, Cubillos I, Marchant J, Panjwani N. Biochemical characterization and functional studies of Acanthamoeba mannose-binding protein. Infect Immun. 2005;73:5775-81.

19. Tachibana H, Cheng XJ, Kobayashi S, Okada Y, Itoh J, Takeuchi T. Primary structure, expression and localization of two intermediate subunit lectins of Entamoeba dispar that contain multiple CXXC motifs. Parasitol. 2007;134: 1989-99.

20. Man S, Fu Y, Guan Y, Feng M, Qiao K, Li X, et al. Evaluation of a major surface antigen of Babesia microti merozoites as a vaccine candidate against Babesia infection. Front Microbiol. 2017;8:2545.

21. Westrop GD, Goodall G, Mottram JC, Coombs GH. Cysteine biosynthesis in Trichomonas vaginalis involves cysteine synthase utilizing O-phosphoserine. J Biol Chem. 2006:281:25062-75.

22. Gaitonde MK. A spectrophotometric method for the direct determination of cysteine in the presence of other naturally occurring amino acids. Biochem J. 1967;104:627-33.

23. Snell K, Walker DG. Regulation of hepatic L-serine dehydratase and L-serinepyruvate aminotransferase in the developing neonatal rat. Biochem J. 1974; 144:519-31.

24. Wu D, Qiao K, Feng M, Fu Y, Cai J, Deng Y, et al. Apoptosis of Acanthamoeba castellanii trophozoites induced by oleic acid. J Eukaryot Microbiol. 2018:65: 191-9.

25. Hell $\mathrm{R}$, Hillebrand $\mathrm{H}$. Plant concepts for mineral acquisition and allocation. Curr Opin Biotechnol. 2001;12:161-8.

26. Nozaki T, Asai T, Sanchez LB, Kobayashi S, Nakazawa M, Takeuchi T. Characterization of the gene encoding serine acetyltransferase, a regulated enzyme of cysteine biosynthesis from the protist parasites Entamoeba histolytica and Entamoeba dispar regulation and possible function of the cysteine biosynthetic pathway in Entamoeba. J Biol Chem. 1999;274:3244552

27. Bonner ER, Cahoon RE, Knapke SM, Jez JM. Molecular basis of cysteine biosynthesis in plants: structural and functional analysis of O-acetylserine sulfhydrylase from Arabidopsis thaliana. J Biol Chem. 2005;280:38803-13.

28. Oda T, Miyajima H, Suzuki Y, Ito T, Yokota S, Hoshino M, et al. Purification and characterization of the active serine: pyruvate aminotransferase of rat liver mitochondria expressed in Escherichia coli. J Biochem. 1989;106:460-7.

29. Snell K, Weber G. Enzymic imbalance in serine metabolism in rat hepatomas. Biochem J. 1986:233:617-20.

30. Dzierszinski F, Popescu O, Toursel C, Slomianny C, Yahiaoui B, Tomavo S. The protozoan parasite Toxoplasma gondii expresses two functional plantlike glycolytic enzymes Implications for evolutionary origin of apicomplexans. J Biol Chem. 1999;274:24888-95.

31. Nozaki T, Asai T, Kobayashi S, Ikegami F, Noji M, Saito K, et al. Molecular cloning and characterization of the genes encoding two isoforms of cysteine synthase in the enteric protozoan parasite Entamoeba histolytica. Mol Biochem Parasitol. 1998:97:33-44

32. Sjoerdsma A, Schechter PJ. Chemotherapeutic implications of polyamine biosynthesis inhibition. Clin Pharmacol Ther. 1984;35:287-300.

33. Kitade $Y$, Kojima H, Zulfiqur F, Yabe S, Yamagiwa D, Ito Y, et al. Synthesis of carbocyclic and acyclic nucleosides possessing 2-fluoroadenine derivatives and their inhibitory activities against Plasmodium falciparum SAH hydrolase. Nucleic Acids Res Suppl. 2003;3:5-6.

34. Khan NA. Acanthamoeba: biology and increasing importance in human health. FEMS Microbiol Rev. 2006;30:564-95.

35. Hug LA, Stechmann A, Roger AJ. Phylogenetic distributions and histories of proteins involved in anaerobic pyruvate metabolism in eukaryotes. Mol Biol Evol. 2010;27:311-24.

36. Alves D, Alves LM, da Costa TL, de Castro AM, Vinaud MC. Anaerobic metabolism in T4 Acanthamoeba genotype. Curr Microbiol. 2017;74:685-90.

37. Zikova A, Hampl V, Paris Z, Tyc J, Lukes J. Aerobic mitochondria of parasitic protists: diverse genomes and complex functions. Mol Biochem Parasitol. 2016:209:46-57.

38. Leger MM, Gawryluk RM, Gray MW, Roger AJ. Evidence for a hydrogenosomal-type anaerobic ATP generation pathway in Acanthamoeba castellanii. PLoS One. 2013;8:e69532.

39. Aqeel $Y$, Siddiqui $R$, Farooq M, Khan NA. Anaerobic respiration: in vitro efficacy of nitazoxanide against mitochondriate Acanthamoeba castellanii of the T4 genotype. Exp Parasitol. 2015:157:170-6.

40. Bouyer S, Rodier MH, Guillot A, Hechard Y. Acanthamoeba castellanii: proteins involved in actin dynamics, glycolysis, and proteolysis are regulated during encystation. Exp Parasitol. 2009;123:90-4. 
41. Tomavo $\mathrm{S}$. The differential expression of multiple isoenzyme forms during stage conversion of Toxoplasma gondii: an adaptive developmental strategy. Int J Parasitol. 2001;31:1023-31.

42. Jeelani G, Sato D, Husain A, Escueta-de Cadiz A, Sugimoto M, Soga T, et al. Metabolic profiling of the protozoan parasite Entamoeba invadens revealed activation of unpredicted pathway during encystation. PLoS One. 2012; e37740

43. Moon EK, Hong Y, Chung DI, Goo YK, Kong HH. Down-regulation of cellulose synthase inhibits the formation of endocysts in Acanthamoeba. Korean J Parasitol. 2014;52:131-5.

44. Dudley R, Alsam S, Khan NA. Cellulose biosynthesis pathway is a potential target in the improved treatment of Acanthamoeba keratitis. Appl Microbiol Biotechnol. 2007;75:133-40

45. Alsam S, Sissons J, Jayasekera S, Khan NA. Extracellular proteases of Acanthamoeba castellanii (encephalitis isolate belonging to T1 genotype) contribute to increased permeability in an in vitro model of the human blood-brain barrier. J Infect. 2005:51:150-6.

Ready to submit your research? Choose BMC and benefit from:

- fast, convenient online submission

- thorough peer review by experienced researchers in your field

- rapid publication on acceptance

- support for research data, including large and complex data types

- gold Open Access which fosters wider collaboration and increased citations

- maximum visibility for your research: over $100 \mathrm{M}$ website views per year

At $\mathrm{BMC}$, research is always in progress.

Learn more biomedcentral.com/submissions 